\title{
Continuous Finite Elements in Space and Time for the Heat Equation
}

\author{
By A. K. Aziz* and Peter Monk** \\ Dedicated to Professor Eugene Isaacson on the occasion of his 70 th birthday
}

\begin{abstract}
In this paper we shall analyze a new variational method for approximating the heat equation using continuous finite elements in space and time. In the special case of linear elements in time the method reduces to the Crank-Nicolson Galerkin method with time-averaged data. Using higher-order finite elements in time, we obtain a new class of time stepping methods related to collocating the standard spatial Galerkin differential equations in time at the Gauss-Legendre points. Again the data enters via suitable time averages. We present error estimates and the results of some numerical experiments.
\end{abstract}

1. Introduction. In this paper we shall analyze a variational method for approximating parabolic problems using continuous finite elements in space and time. For simplicity, we shall apply the method to the following initial-boundary value problem for the heat equation. Let $\Omega$ be a polygonal domain in $\mathbf{R}^{2}$ with boundary $\partial \Omega$, let $T>0$, and let $u(\mathbf{x}, t)$ satisfy

$$
\begin{array}{ll}
u_{t}=\Delta u+f & \forall(\mathbf{x}, t) \in \Omega \times(0, T), \\
u=0 & \forall(\mathbf{x}, t) \in \partial \Omega \times[0, T], \\
u=u_{0} & \forall \mathbf{x} \in \Omega \text { and } t=0,
\end{array}
$$

where $f(\mathbf{x}, t)$ and $u_{0}(\mathbf{x})$ are given functions. The extension of our method to more general linear problems follows in obvious ways.

To formulate the finite element method, let $S_{h}^{p}(\Omega) \subset H_{0}^{1}(\Omega)$ be a finite element space of continuous piecewise $p$ th degree polynomials on $\Omega$ with mesh parameter $h$ (we shall give more details of the finite element spaces used in Section 2). Let $0=t_{0}<t_{1}<t_{2}<\cdots<t_{N}=T$ be a partition of $[0, T]$, and let $S_{k}^{q}([0, T])$ be a finite element space on this partition consisting of continuous piecewise $q$ th degree polynomials in time $\left(k=\max _{1 \leq i \leq N}\left|t_{i}-t_{i-1}\right|\right)$. Then define $W_{h k}$ to be the tensor product space

$$
W_{h k}=S_{h}^{p}(\Omega) \otimes S_{k}^{q}([0, T])
$$

If we let $(\cdot, \cdot)$ denote the inner product on $L^{2}(\Omega)$, our finite element method for approximating (1.1) is to find $u^{h k} \in W_{h k}$ such that

$$
u^{h k}=P^{x} u_{0} \quad \text { at } t=0
$$

Received April 8, 1987; revised October 26, 1987 and April 28, 1988.

1980 Mathematics Subject Classification (1985 Revision). Primary 65N30.

Key words and phrases. Galerkin method, heat equation, error estimates.

* Research supported in part by IR funds of NSWC.

${ }^{* *}$ Research supported in part by grants from the AFOSR and the NSF. 
where $P^{x}$ is the elliptic projection onto $S_{h}^{p}(\Omega)$ (see Section 2), and

$$
\int_{0}^{T}\left(u_{t}^{h k}, v_{t}^{h k}\right)+\left(\nabla u^{h k}, \nabla v_{t}^{h k}\right) d t=\int_{0}^{T}\left(f, v_{t}^{h k}\right) d t \quad \forall v^{h k} \in W_{h k} .
$$

The finite element solution $u^{h k}$ can be computed by marching through successive time levels. To see this, let $P^{q}(J)$ denote the set of polynomials of degree $q$ on an interval $J$; then for $n=0,1,2, \ldots, N-1$ we can compute $u^{h k}$ on $\left[t_{n}, t_{n+1}\right]$ as the unique solution (see Section 3 ) of

$$
\begin{aligned}
\int_{t_{n}}^{t_{n+1}}\left(u_{t}^{h k}, w^{h k}\right)+\left(\nabla u^{h k}, \nabla w^{h k}\right) d t= & \int_{t_{n}}^{t_{n+1}}\left(f, w^{h k}\right) d t \\
& \forall w^{h k} \in S_{h}^{p}(\Omega) \otimes P^{q-1}\left(\left[t_{n}, t_{n+1}\right]\right)
\end{aligned}
$$

with $u^{h k}$ at $t=t_{n}$ fixed by continuity (or initial conditions if $n=0$ ). Clearly, a solution of (1.4) for $n=0,1, \ldots, N-1$ is also a solution of (1.3) and vice versa. Thus, although $u^{h k}$ is continuous in space and time, we can solve the finite element problem (1.3) by marching in time. Equation (1.4) also shows that the finite element method proposed in (1.3) can be viewed as a Petrov-Galerkin method for approximating the heat equation, in which the trial function $u^{h k}$ is continuous in space and time, whereas the test function $w^{h k}=v_{t}^{h k}$ is discontinuous in time and continuous in space.

Many finite element methods for the heat equation have been proposed and analyzed in the literature (cf. [18]). A common approach is to first apply the Galerkin method in space to reduce (1.1) to a system of ordinary differential equations. In that approach, called the method of lines, we seek $u^{h}(t) \in S_{h}^{p}(\Omega)$ such that

$$
\left(u_{t}^{h}, v^{h}\right)+\left(\nabla u^{h}, \nabla v^{h}\right)=\left(f, v^{h}\right) \quad \forall v^{h} \in S_{h}^{p}(\Omega) .
$$

A suitable method is then applied to integrate this system of ordinary differential equations. One interesting scheme results when the trapezoidal rule is applied to (1.5) and this fully discrete method is called the Crank-Nicolson Galerkin method (cf. [20] and [18]). In contrast to the method of lines described above, we use finite elements to discretize in space and time simultaneously. However, if we use piecewise linear functions in time (i.e., $q=1$ ) in (1.3), we obtain a version of the Crank-Nicolson Galerkin method in which the data $f$ enters as time averages. Thus our analysis yields an alternative proof of convergence for a Crank-Nicolson type method. To understand the method when $q>1$, consider discretizing the homogeneous problem (1.1) with $f \equiv 0$. If we apply $q$-point Gauss-Legendre quadrature on each subinterval in time, we evaluate the time integrals in (1.4) exactly. Furthermore, by a suitable choice of $w^{h k}$, we can show that (1.5) is exactly satisfied at the Gauss-Legendre points in time. Hence, for the homogeneous problem, our method is exactly equivalent to collocating (1.5) at the Gauss-Legendre points on each subinterval in time. Furthermore, for this linear problem $q$-point Gauss-Legendre collocation is known to be equivalent (in the sense of giving the same nodal values of the solution) to using the $q$ th diagonal Padé approximation (cf. [9]). For the inhomogeneous problem, our method differs from Gauss-Legendre point collocation in that the data $f$ appears as suitable time averages. The Gauss-Legendre collocation methods can be thought of as a good quadrature method applied to implement our 
method. The choice of a $q$-point Gauss-Legendre quadrature rule for integration in time is not the only possibility, however. One could approximate the time integrals in (1.4) by a $q$-point Radau quadrature on each subinterval $\left[t_{n}, t_{n+1}\right]$ with $t_{n+1}$ chosen as a quadrature point. Again, for the linear homogeneous problem, we obtain a collocation method for (1.5) and this method is equivalent to using the $(q, q-1)$ subdiagonal Padé approximation (cf. [8]). In this case the integrals in time are not computed exactly by the quadrature formula, and there is a loss of accuracy in the time step.

The use of continuous finite element methods to discretize time-dependent problems has been analyzed before for ordinary differential equations. Hulme [9], [8] has investigated continuous time Galerkin methods for ordinary differential equations, and shown how they relate to collocation methods. He has proved convergence for the associated collocation schemes applied to nonlinear ordinary differential equations. For hyperbolic problems, Winther [21] has analyzed exactly the time stepping scheme we propose when applied to a system of first-order hyperbolic problems. As pointed out above, our variational method is closely related to Gauss-Legendre point collocation methods for the heat equation, and these methods have been analyzed by Douglas and Dupont [3] in the context of collocation in space and time for one space dimension problems. Our work is the first analysis of the continuous time Galerkin method applied to the heat equation.

The use of finite elements in space and time for parabolic problems is not new. A significant body of work has analyzed the use of discontinuous in time finite element methods (cf. [6] and [10], and the references contained in those works). In particular, the discontinuous in time Galerkin methods of Eriksson, Johnson and Thomée [6] generalize the implicit Euler scheme for time stepping the heat equation, using as data time averages of $f$. In general, when applied to the homogeneous problem, the discontinuous Galerkin method is equivalent to using a subdiagonal Padé approximation to discretize (1.5) in time. Thus the smoothing properties of the below-diagonal Padé approximation are built into the method. The discontinuous Galerkin method may be useful in designing adaptive schemes, and Eriksson and Johnson [5] have examined this possibility. It is hoped that our method will also provide a basis for an adaptive scheme; however, since the Crank-Nicolson method is known to behave poorly on problems with rough solutions (and this is presumably also true of the higher-order methods), the applicability to adaptive methods is not certain.

Other finite element methods using elements that are continuous in time have been proposed in the past. For example Lesaint and Raviart [13] proposed a collocation method for solving the heat equation rewritten as a first-order system. Jamet [11] has also investigated methods on variable grids. However, we believe that (1.3) is a new Galerkin formulation for the heat equation, and that the resulting methods are novel.

The plan for the remainder of this paper is as follows. In Section 2 we will summarize notation and describe in detail the finite element spaces used. In Section 3 we shall prove some global error estimates for the solution of (1.4) and show the method is of optimal order. Our analysis will draw on the work of Falk and 
Richter [7] who have analyzed a continuous finite element method for the transport equation, which motivated our formulation. We have also used ideas from [18].

We should comment on the smoothness and convexity requirements of our theorems. We have assumed $\Omega$ to be a polygonal domain in order to avoid the increased complexity of dealing with variational crimes which arise when approximating Dirichlet problems on a smooth domain. Unfortunately, on polygonal domains, smooth data may not give rise to smooth solutions, because corners induce singularities. Thus we have not attempted to relate the smoothness required by our estimates to properties of the data. Of greater concern is that we cannot apply duality arguments on a general polygonal domain, and so in some theorems we have to assume the domain is convex. It is our opinion that the clarity of exposition possible with a polygonal domain outweighs these difficulties.

The fact that our time stepping method is equivalent to a diagonal Padé approximation when applied to the homogeneous problem, suggests that the method should converge with time discretization error proportional to $k^{2 q}$ at the time step points $t_{n}$, and in Section 4 we shall prove such a superconvergence result. The proof of superconvergence uses some special projections introduced by Winther [21], which are related to the quasi-projections of Douglas, Dupont and Wheeler [4]. Finally, in Section 5 we will present some limited numerical results concerning superconvergence estimates and the utility of higher-order methods for smooth problems.

2. Notation and Finite Element Spaces. First let us define some notation to be used in the remainder of this paper. Let $\Omega$ be a bounded polygonal domain in the plane, and let $L^{2}(\Omega)$ be the standard space of square integrable functions on $\Omega$ with inner product $(\cdot, \cdot)$ defined by

$$
(u, v)=\int_{\Omega} u(\mathbf{x}) v(\mathbf{x}) d \mathbf{x}
$$

and norm $\|u\|_{0}=(u, u)^{1 / 2}$. For integral $s \geq 0, H^{s}(\Omega)$ is the completion of $C^{\infty}(\bar{\Omega})$ in the norm

$$
\|\phi\|_{s}^{2}=\sum_{|\alpha| \leq s}\left\|D^{\alpha} u\right\|_{0}^{2}
$$

where $\alpha=(i, j),|\alpha|=i+j$, and $D^{\alpha}=\left(\partial / \partial x_{1}\right)^{i}+\left(\partial / \partial x_{2}\right)^{j}$. For nonintegral $s$, $H^{s}(\Omega)$ is defined by interpolation (cf. [14]), and $H_{0}^{1}(\Omega)=\left\{u \in H^{1}(\Omega) \mid u=0\right.$ on $\partial \Omega$ \}. We shall also need to use anisotropic Sobolev spaces. Let $J$ be an interval; then $C^{\infty}\left(\bar{J} ; H^{s}(\Omega)\right)$ denotes the set of infinitely differentiable functions from $J$ into $H^{s}(\Omega)$ for which all derivatives have continuous extensions to $\bar{J}$. For nonnegative $r$ and $s$ we define $H^{r}\left(J ; H^{s}(\Omega)\right)$ to be the completion of $C^{\infty}\left(\bar{J} ; H^{s}(\Omega)\right)$ in the norm

$$
\left(\|u\|_{H^{r}\left(J ; H^{s}(\Omega)\right)}\right)^{2}=\sum_{j=0}^{r} \int_{J}\left\|\frac{\partial^{j}}{\partial t^{j}} u(\cdot, t)\right\|_{s}^{2} d t
$$

and we define

$$
H^{r, s}(\Omega \times J)=H^{0}\left(J ; H^{r}(\Omega)\right) \cap H^{s}\left(J ; H^{0}(\Omega)\right)
$$

with norm

$$
\left(\|u\|_{r, s}^{J}\right)^{2}=\|u\|_{H^{0}\left(J ; H^{r}(\Omega)\right)}^{2}+\|u\|_{H^{s}\left(J ; H^{0}(\Omega)\right)}^{2} .
$$


For each nonnegative $r$ and $s, H^{r, s}(\Omega \times J)$ is a Hilbert space defined by interpolation in $r$ and $s$ [15]. Further, let

$$
H_{0}^{1,1}=\left\{u \in H^{1,1}(\Omega \times(0, T)): u=0 \text { on } \partial \Omega \times(0, T)\right\} .
$$

In addition, in the section of the paper concerning superconvergence, we shall also use the spaces $H^{s}\left(\Omega ; H^{r}(0, T)\right)$ which are defined analogously to the spaces discussed above.

Properties of the spaces defined above, including trace theorems and theorems concerning the existence of mixed derivatives, are proved in [14] and [15] when $\Omega$ is smooth. For polygonal domains, the existence of mixed derivatives and extension theorems are proved in [17]. The use of anisotropic spaces on polygonal domains for parabolic problems is discussed in [12].

Now let us describe the finite element spaces used in this paper. Let $\tau_{h}(\Omega)$ denote a regular triangulation of $\Omega$ satisfying the usual finite element geometric constraints [2], where $h$ is the maximum diameter of the triangles in $\tau_{h}(\Omega)$. Let $S_{h}^{p}(\Omega) \subset H_{0}^{1}(\Omega)$ be the set of all continuous piecewise $p$ th degree polynomials on $\tau_{h}(\Omega)$ that vanish on $\partial \Omega$. We remark that (1.3) can also be applied using finite element spaces based on a quadrilateral tesselation of $\Omega$, and the estimates we shall prove in Sections 3 and 4 also hold in that case.

Now define $P^{x}: H_{0}^{1}(\Omega) \rightarrow S_{h}^{p}(\Omega)$ to be the elliptic projection, so that if $u \in$ $H_{0}^{1}(\Omega), P^{x} u \in S_{h}^{p}(\Omega)$ satisfies

$$
\left(\nabla P^{x} u, \nabla \phi_{h}\right)=\left(\nabla u, \nabla \phi_{h}\right) \quad \forall \phi_{h} \in S_{h}^{p}(\Omega) .
$$

By virtue of the regularity of the triangulation it is well known (cf. [1], [2]) that $P^{x}$ has the following approximation properties. If $u \in H_{0}^{1}(\Omega) \cap H^{r}(\Omega)$, then

$$
\left\|u-P^{x} u\right\|_{s} \leq C h^{r-s}\|u\|_{r}
$$

for $1 \leq r \leq p+1$ and $s=1$. In addition, if $\Omega$ is convex, this estimate holds for $0 \leq s \leq 1$.

$P^{x}$ can be extended to functions of $\mathbf{x}$ and $t$ in an $L^{2}$ sense. Thus we define the extended projection $P^{x}: H_{0}^{1,1}(\Omega \times(0, T)) \rightarrow S_{h}^{p}(\Omega) \otimes L^{2}(0, T)$ by

$$
\int_{0}^{T}\left(\nabla P^{x} u, \nabla \phi_{h}\right) d t=\int_{0}^{T}\left(\nabla u, \nabla \phi_{h}\right) d t \quad \forall \phi_{h} \in S_{h}^{p}(\Omega) \otimes L^{2}(0, T) .
$$

Error estimates for the extended projection are given later in Lemma 2.2 and follow from the approximation properties of $P^{x}$ given by (2.3). Note, in particular, if $u$ is smooth enough that $u(\cdot, t) \in H_{0}^{1}(\Omega)$ for each $t$, we can define $P^{x} u$ pointwise in time, and error estimates for the extended projection follow from (2.3).

We shall use the finite element solution of the Dirichlet problem for Laplace's equation on $\Omega$. Let $T: H^{-1}(\Omega) \rightarrow H_{0}^{1}(\Omega)$ be such that if $g \in H^{-1}(\Omega)$, then $T g \in H_{0}^{1}(\Omega)$ satisfies

$$
(\nabla T g, \nabla \phi)=(g, \phi) \quad \forall \phi \in H_{0}^{1}(\Omega) .
$$

Let $T_{h}$ be the finite element approximation to $T$ defined as follows. $T_{h}: H^{-1}(\Omega) \rightarrow$ $S_{h}^{p}(\Omega)$, such that if $g \in H^{-1}(\Omega)$ then $T_{h} g \in S_{h}^{p}(\Omega)$ satisfies

$$
\left(\nabla T_{h} g, \nabla \phi_{h}\right)=\left(g, \phi_{h}\right) \quad \forall \phi_{h} \in S_{h}^{p}(\Omega) .
$$


The approximation properties of $T_{h}$ are also well known and follow from the properties of $P^{x}$ (cf. [1]) since $T_{h}=P^{x} T$. Of course, $T_{h}$ can also be extended to apply to functions in $H^{0,0}(\Omega \times(0, T))$ in an $L^{2}$ sense (in the same way as for $\left.P^{x}\right)$. Furthermore, $T_{h}$ is a symmetric operator, and $T_{h}$ satisfies the following a priori bound

$$
0 \leq\left(g, T_{h} g\right) \leq C\|g\|_{-1}^{2} \quad \forall g \in H^{-1}(\Omega) .
$$

The left-hand inequality follows from the definition of $T_{h}$. The right-hand inequality is proved using the a priori estimate $\|T g\|_{1} \leq C\|g\|_{-1}$ and the approximation property $\left\|\left(T-T_{h}\right) g\right\|_{1} \leq C\|T g\|_{1}$. Note also that $T_{h}$ is invertible on $S_{h}^{p}(\Omega)$, since if $T_{h} u^{h}=.0$ for some $u^{h} \in S_{h}^{p}(\Omega)$, then $\left(u^{h}, u^{h}\right)=\left(\nabla T_{h} u^{h}, \nabla u^{h}\right)=0$ and hence $u^{h}=0$.

Next we discuss finite element subspaces in time. Let $0=t_{0}<t_{1}<t_{2}<$ $\cdots<t_{N}=T$ be a partition of $[0, T]$ and let $k=\max _{1 \leq n \leq N}\left|t_{n}-t_{n-1}\right|$. Then define $S_{k}^{q}([0, T]) \subset H^{1}(0, T)$ to be the set of all continuous piecewise $q$ th degree polynomials on this partition. Suppose $J_{n}=\left[0, t_{n}\right], 0<n$; then $S_{k}^{q}\left(J_{n}\right)$ is the restriction to $J_{n}$ of functions in $S_{k}^{q}([0, T])$.

We also need to consider projections in time. Let $P^{t}: H^{1}(0, T) \rightarrow S_{k}^{q}([0, T])$ be the one-dimensional projection such that if $w \in H^{1}(0, T)$, then $P^{t} w \in S_{k}^{q}([0, T])$ satisfies $P^{t} w(0)=w(0)$ and

$$
\int_{0}^{T}\left(P^{t} w\right)_{t} \phi_{t}^{k} d t=\int_{0}^{T} w_{t} \phi_{t}^{k} d t \quad \forall \phi^{k} \in S_{k}^{q}([0, T]) .
$$

By standard techniques, we can see that $P^{t}$ satisfies the estimate

$$
\left\|w-P^{t} w\right\|_{H^{s}(0, T)} \leq C k^{r-s}\|w\|_{H^{r}(0, T)}
$$

for $-q+1 \leq s \leq 1 \leq r \leq q+1$ with $C$ independent of $w$ but dependent on $T$. Again, we can extend $P^{t}$ to apply to functions of $\mathbf{x}$ and $t$ in an $L^{2}$ sense. Thus we define $P^{t}: H^{0,1}(\Omega \times(0, T)) \rightarrow L^{2}(\Omega) \otimes S_{k}^{q}([0, T])$ by

$$
\int_{0}^{T}\left(\left(P^{t} w\right)_{t}, \phi_{t}^{k}\right) d t=\int_{0}^{T}\left(w_{t}, \phi_{t}^{k}\right) d t \quad \forall \phi^{k} \in L^{2}(\Omega) \otimes S_{k}^{q}([0, T])
$$

with the initial condition $\left(\left(P^{t} u\right)(\cdot, 0), \phi\right)=(u(\cdot, 0), \phi) \forall \phi \in L^{2}(\Omega)$ (the trace of $u \in H^{0,1}(\Omega \times(0, T))$ is defined at $t=0, \mathrm{cf}$. [15]). Error estimates for the extended operator are given in Lemma 2.2 .

Note that by taking $\phi^{k}=t$ for $0 \leq t \leq t_{n}$ and $\phi^{k}=t_{n}$ for $t>t_{n}$ in (2.7), we conclude that

$$
P^{t} w\left(t_{n}\right)=w\left(t_{n}\right)
$$

(in particular, if $q=1, P^{t}$ is just the interpolant). Thus the projection of $w$ onto $S_{k}^{q}\left(\left[0, t_{n}\right]\right)$ defined similarly to $(2.7)$ (with $T$ replaced by $t_{n}$ ) is just the restriction of $P^{t} w$ to $\left[0, t_{n}\right]$ and satisfies estimate $(2.8)$ with $[0, T]$ replaced by $\left[0, t_{n}\right]$ (and $C$ independent of $n$ but dependent on $T$ ).

Now let $W_{h k}=S_{h}^{p}(\Omega) \otimes S_{k}^{q}([0, T])$. We will need some approximation properties of this space. First we note that operators in space and time commute. 
LEMMA 2.1. Suppose $u \in H^{2,2}(\Omega \times(0, T))$; then

$$
\begin{aligned}
\left(P^{x} u\right)_{t} & =P^{x}\left(u_{t}\right), \\
\nabla P^{t} u & =P^{t} \nabla u, \\
P^{x} P^{t} u & =P^{t} P^{x} u, \\
T_{h} P^{t} u & =P^{t} T_{h} u .
\end{aligned}
$$

Proof. The regularity assumed for $u$ guarantees that $\nabla u_{t}$ is well defined (cf. [17]). The results then follow from the linearity of the operators, and we will give a proof only of the first result. Let $\phi^{h} \in S_{h}^{p}(\Omega) \otimes H^{1}(0, T)$ be an arbitrary function with $\phi^{h}(\cdot, 0)=\phi^{h}(\cdot, T)=0$; then

$$
\begin{aligned}
\int_{0}^{T}\left(\nabla P^{x} u_{t}, \nabla \phi^{h}\right) d t & =\int_{0}^{T}\left(\nabla u_{t}, \nabla \phi^{h}\right) d t=-\int_{0}^{T}\left(\nabla u, \nabla \phi_{t}^{h}\right) d t \\
= & -\int_{0}^{T}\left(\nabla P^{x} u, \nabla \phi_{t}^{h}\right) d t=\int_{0}^{T}\left(\nabla\left(P^{x} u\right)_{t}, \nabla \phi^{h}\right) d t
\end{aligned}
$$

thus $P^{x} u_{t}=P^{x}\left(P^{x} u\right)_{t}$ but $\left(P^{x} u\right)_{t} \in S_{h}^{p}(\Omega) \otimes L^{2}(0, T)$, and the result is proved. The remaining results can be proved similarly, and we shall not provide details here.

Lemma 2.1 allows us to prove the following approximation results:

LEMMA 2.2. Let $P^{x}$ and $P^{t}$ be defined in the extended sense by (2.4) and (2.9), and let $J_{n}=\left(0, t_{n}\right), 1 \leq n \leq N$. Then there is a constant $C$ independent of $n$ such that the following hold.

1. Suppose $v \in H^{0, s}\left(\Omega \times J_{n}\right)$. Then for $-q+1 \leq r \leq 1 \leq s \leq q+1$,

$$
\int_{\Omega} \sum_{m=0}^{n-1}\left\|v-P^{t} v\right\|_{H^{r}\left(t_{m}, t_{m+1}\right)}^{2} d x \leq C k^{2(s-r)}\left(\|v\|_{0, s}^{J_{n}}\right)^{2} .
$$

2. Suppose $v \in H^{2,1}\left(\Omega \times J_{n}\right) \cap H_{0}^{1,1}\left(\Omega \times J_{n}\right)$ and $v(\cdot, t) \in H^{p+1}(\Omega)$ for all $t$; then for $s=1$ in general, or for $0 \leq s \leq 1$ if $\Omega$ is convex,

$$
\left\|\left(v-P^{x} v\right)(\cdot, t)\right\|_{s} \leq C h^{p+1-s}\|v(\cdot, t)\|_{p+1} .
$$

3. Suppose $\left.v \in H^{r, 0}\left(\Omega \times J_{n}\right) \cap H_{0}^{1,1} \Omega \times J_{n}\right)$ and $\Omega$ is convex. Then for $1 \leq r \leq$ $p+1$,

$$
\left\|P^{x} v-v\right\|_{0,0}^{J_{n}} \leq C h^{r}\|v\|_{r, 0}^{J_{n}}
$$

4. Suppose $u \in H^{0, q+1}\left(\Omega \times J_{n}\right) \cap H_{0}^{1,1}\left(\Omega \times J_{n}\right)$ and $u_{t} \in H^{p+1,0}\left(\Omega \times J_{n}\right) \cap$ $H_{0}^{1,1}\left(\Omega \times J_{n}\right)$ and $\Omega$ is convex. Then

$$
\left\|\left(u-P^{x} P^{t} u\right)_{t}\right\|_{0,0}^{J_{n}} \leq C\left\{h^{p+1}\left\|u_{t}\right\|_{p+1,0}^{J_{n}}+k^{q}\|u\|_{0, q+1}^{J_{n}}\right\} .
$$

Proof. Each of parts (1), (3) and (4) are proved in the same way, so we will give details only for part (1). We start by proving the estimate for a smooth function $u \in C^{\infty}\left(\Omega \times J_{n}\right)$. Using (2.8) together with the fact that $P^{t}$ is a local projection 
on each subinterval in time,

$$
\begin{aligned}
& \sum_{m=0}^{n-1} \int_{\Omega}\left\|v-P^{t} v\right\|_{H^{r}\left(t_{m}, t_{m+1}\right)}^{2} d \mathbf{x} \\
& \quad=\sum_{m=0}^{n-1} \int_{\Omega}\left\|v(\mathbf{x}, \cdot)-\left(P^{t} v\right)(\mathbf{x}, \cdot)\right\|_{H^{r}\left(t_{m}, t_{m+1}\right)}^{2} d \mathbf{x} \\
& \quad \leq \sum_{m=0}^{n-1} \int_{\Omega} C k^{2(s-r)}\|v(\mathbf{x}, \cdot)\|_{H^{s}\left(t_{m}, t_{m+1}\right)}^{2} d \mathbf{x} \\
& \quad \leq C k^{2(s-r)}\left(\|v\|_{0, s}^{J_{n}}\right)^{2}
\end{aligned}
$$

Now consider a sequence of smooth functions $\left\{v_{i}\right\}$ converging to a function $v$ in $H^{0, s}\left(\Omega \times J_{n}\right)$ and apply the above estimates to each $v_{i}$. Since the estimate holds for each $i$, it holds in the limit. This completes the proof of (1).

Part (2) follows from (2.3) since the regularity assumptions ensure $v(\cdot, t) \in$ $H_{0}^{1}(\Omega)$ for each $t$. Part (3) is proved in a fashion similar to part (1). Part (4) is also proved for a smooth function first. If $v \in C^{\infty}\left(\Omega \times J_{n}\right)$ with $v=0$ on $\partial \Omega$, then using the commutation properties of $P^{x}$ and $P^{t}$ in Lemma 2.1,

$$
\begin{aligned}
\left\|\left(v-P^{x} P^{t} v\right)_{t}\right\|_{0,0}^{J_{n}} \leq & \left\|\left(v-P^{t} v\right)_{t}\right\|_{0,0}^{J_{n}}+\left\|\left(v-P^{x} v\right)_{t}\right\|_{0,0}^{J_{n}} \\
& +\left\|\left(\left(v-P^{x} v\right)-P^{t}\left(v-P^{x} v\right)\right)_{t}\right\|_{0,0}^{J_{n}} .
\end{aligned}
$$

Application of (2.3) and (2.8) pointwise proves the estimate if $v$ is smooth. A limiting argument similar to that given for part (1) completes the proof of part (4).

3. Global Error Estimates. In this section we will prove existence, uniqueness, and some global error estimates for the numerical scheme given by (1.3).

THEOREM 3.1. For any $u_{0} \in H_{0}^{1}(\Omega)$ and $f \in H^{0,0}(\Omega \times(0, T))$ there exists a unique solution $u^{\text {hk }}$ satisfying (1.2) and (1.3). Furthermore, the following stability estimate holds for $1 \leq n \leq N$ with constant $C$ independent of $n$ and $N$ :

$$
\left(\left\|T_{h}^{1 / 2} u_{t}^{h k}\right\|_{0,0}^{\left(0, t_{n}\right)}\right)^{2}+\left\|u^{h k}\left(\cdot, t_{n}\right)\right\|_{0}^{2} \leq C\left\{\left(\left\|T_{h}^{1 / 2} f\right\|_{0,0}^{\left(0, t_{n}\right)}\right)^{2}+\left\|P^{x} u_{0}\right\|_{0}^{2}\right\} .
$$

Proof. As discussed in the introduction, problem (1.2)-(1.3) is equivalent to the problem of finding $u^{h k} \in W_{h k}$ such that

$$
u^{h k}(\cdot, 0)=P^{x} u_{0}
$$

and

$$
\begin{aligned}
\int_{t_{m}}^{t_{m+1}}\left(u_{t}^{h k}, w^{h k}\right)+\left(\nabla u^{h k}, \nabla w^{h k}\right) d t= & \int_{t_{m}}^{t_{m+1}}\left(f, w^{h k}\right) d t \\
& \forall w^{h k} \in S_{h}^{p}(\Omega) \otimes P^{q-1}\left(\left[t_{m}, t_{m+1}\right]\right)
\end{aligned}
$$

for $m=0,1,2, \ldots, N-1$. For each $m, u^{h k}$ is determined at $t_{m}$ (by $u_{0}$ if $m=0$, and by continuity if $m>0$ ). Thus, for each $m$, the number of equations given by (3.2) and the number of degrees of freedom (just $\left|S_{h}^{p}(\Omega)\right| \cdot q$ where $\left|S_{h}^{p}(\Omega)\right|$ is the number of degrees of freedom in $\left.S_{h}^{p}(\Omega)\right)$ are equal, and it suffices to prove uniqueness. 
Uniqueness can be proved via the stability result in the theorem. To prove stability, consider (3.1)-(3.2), and take $w^{h k}=T_{h} u_{t}^{h k}$. Then using the definition of $T_{h}$ in Section 2 ,

$$
\int_{t_{m}}^{t_{m+1}}\left(u_{t}^{h k}, T_{h} u_{t}^{h k}\right)+\left(u^{h k}, u_{t}^{h k}\right) d t=\int_{t_{m}}^{t_{m+1}}\left(f, T_{h} u_{t}^{h k}\right) d t
$$

Adding this estimate for $m=0,1, \ldots, n-1$, and using the definition of $\|\cdot\|_{0,0}^{J}$ given in Section 2, we obtain

$$
\left(\left\|T_{h}^{1 / 2} u_{t}^{h k}\right\|_{0,0}^{\left(0, t_{n}\right)}\right)^{2}+\left\|u^{h k}\left(\cdot, t_{n}\right)\right\|_{0}^{2} \leq C\left\{\left(\left\|T_{h}^{1 / 2} f\right\|_{0,0}^{\left(0, t_{n}\right)}\right)^{2}+\left\|u^{h k}(\cdot, 0)\right\|_{0}^{2}\right\}
$$

Using the initial condition (3.1) proves the stability estimate.

To prove uniqueness, we apply the stability estimate with $u_{0} \equiv 0$ and $f \equiv 0$, and conclude $u^{h k}\left(\mathbf{x}, t_{n}\right)=0$ for $\mathbf{x} \in \Omega$ and $0 \leq n \leq N$, and $\left\|T_{h}^{1 / 2} u_{t}^{h k}\right\|_{0,0}^{\left(0, t_{n}\right)}=0$. But since $T_{h}$ is invertible on $S_{h}^{p}(\Omega)$ (see Section 2 ), we conclude $u_{t}^{h k} \equiv 0$, and so $u^{h k} \equiv 0$. Hence existence and uniqueness are guaranteed.

Our next theorem guarantees approximability in $H^{1}(\Omega)$ norms:

THEOREM 3.2. Let $u$ solve (1.1), let $u^{h k}$ satisfy (1.2) and (1.3), and let $e(\mathbf{x}, t)$ $=u(\mathbf{x}, t)-u^{h k}(\mathbf{x}, t)$. Then the following estimates hold for $n=1,2, \ldots, N$ :

1. Suppose $u(\cdot, t) \in H^{p+1}(\Omega)$ for $0 \leq t \leq T, \Delta u \in H^{0, q+1}(\Omega \times(0, T))$, and $u_{t} \in H^{p, 0}(\Omega \times(0, T))$. Then

$$
\left\|e\left(\cdot, t_{n}\right)\right\|_{1} \leq C\left\{k^{q+1}\|\Delta u\|_{0, q+1}^{\left(0, t_{n}\right)}+h^{p}\left(\left\|u_{t}\right\|_{p, 0}^{\left(0, t_{n}\right)}+\left\|u\left(\cdot, t_{n}\right)\right\|_{p+1}\right)\right\}
$$

2. Suppose $\Delta u \in H^{0, q}(\Omega \times(0, T)), u \in H^{0, q+1}(\Omega \times(0, T)), u_{t} \in H^{p+1,0}(\Omega \times(0, T))$ and $\Omega$ is convex. Then

$$
\left\|e_{t}\right\|_{0,0}^{\left(0, t_{n}\right)} \leq C\left\{k^{q}\left(\|u\|_{0, q+1}^{\left(0, t_{n}\right)}+\|\Delta u\|_{0, q}^{\left(0, t_{n}\right)}\right)+h^{p+1}\left\|u_{t}\right\|_{p+1,0}^{\left(0, t_{n}\right)}\right\}
$$

Remark. The estimate for $e_{t}$ is of optimal order, since the norm is global. For a general polygonal domain we must replace the term $h^{p+1}$ by a power of $h$ that depends on the nature of the corners of the polygon.

In order to prove this theorem, and a subsequent theorem concerning error in lower norms, we first state and prove the following lemma.

LEMMA 3.1. Let $P^{x}$ and $P^{t}$ be the projections defined in Section 2, and assume $u \in H^{2,2}(\Omega \times(0, T)) \cap H_{0}^{1,1}(\Omega \times(0, T))$. Then for any $\phi \in W_{h k}$,

$$
\begin{gathered}
\int_{0}^{t_{n}}\left(\left(P^{t} P^{x} u-u^{h k}\right)_{t}, \phi_{t}\right)+\left(\nabla\left(P^{t} P^{x} u-u^{h k}\right), \nabla \phi_{t}\right) d t \\
=\int_{0}^{t_{n}}\left(\left(P^{x} u-u\right)_{t}, \phi_{t}\right)-\left(\Delta\left(P^{t} u-u\right), \phi_{t}\right) d t
\end{gathered}
$$


Proof. We use the definitions of $P^{x}$ and $P^{t}$, together with the commutation property of the projections (Lemma 2.1) as follows:

$$
\begin{aligned}
\int_{0}^{t_{n}}( & \left.\left(P^{t} P^{x} u-u^{h k}\right)_{t}, \phi_{t}\right)+\left(\nabla\left(P^{t} P^{x} u-u^{h k}\right), \nabla \phi_{t}\right) d t \\
= & \int_{0}^{t_{n}}\left(\left(P^{t} P^{x} u-P^{t} u\right)_{t}, \phi_{t}\right)+\left(\nabla\left(P^{t} P^{x} u-P^{x} u\right), \nabla \phi_{t}\right) d t \\
& +\int_{0}^{t_{n}}\left(\left(P^{t} u-u^{h k}\right)_{t}, \phi_{t}\right)+\left(\nabla\left(P^{x} u-u^{h k}\right), \nabla \phi_{t}\right) d t \\
= & \int_{0}^{t_{n}}\left(\left(P^{x} u-u\right)_{t}, \phi_{t}\right)+\left(\nabla\left(P^{t} u-u\right), \nabla \phi_{t}\right) d t \\
& +\int_{0}^{t_{n}}\left(\left(u-u^{h k}\right)_{t}, \phi_{t}\right)+\left(\nabla\left(u-u^{h k}\right), \nabla \phi_{t}\right) d t
\end{aligned}
$$

Use of the definition of $u^{h k}(1.3)$ and the equation for $u$, together with integration by parts, completes the proof of this lemma.

Proof of Theorem 3.2. The regularity assumptions on $u$ and $u_{t}$ ensure that $u \in H^{2,2}(\Omega \times(0, T)) \cap H_{0}^{1,1}(\Omega \times(0, T))$, and hence we may apply Lemma 3.1 with $\phi=P^{t} P^{x} u-u^{h k}$. Note by the interpolation property of $P^{t}$ given by $(2.10), \phi(\cdot, 0)=$ $\left(P^{t} P^{x} u-u^{h k}\right)(\cdot, 0)=\left(P^{x} u(\cdot, 0)-P^{x} u_{0}(\cdot)\right)=0$. Using Schwarz's inequality and the arithmetic geometric mean inequality, we obtain

$$
\begin{array}{r}
\left\|\left(P^{t} P^{x} u-u^{h k}\right)_{t}\right\|_{0,0}^{\left(0, t_{n}\right)}+\left\|\nabla\left(P^{t} P^{x} u-u^{h k}\right)\left(\cdot, t_{n}\right)\right\|_{0} \\
\leq C\left(\left\|\left(P^{x} u-u\right)_{t}\right\|_{0,0}^{\left(0, t_{n}\right)}+\left\|\Delta\left(P^{t} u-u\right)\right\|_{0,0}^{\left(0, t_{n}\right)}\right) .
\end{array}
$$

Hence, by the triangle inequality, and noting that, by $(2.10), P^{t} P^{x} u\left(\cdot, t_{n}\right)=$ $P^{x} u\left(\cdot, t_{n}\right)$,

$$
\begin{aligned}
\left\|\nabla\left(u-u^{h k}\right)\left(\cdot, t_{n}\right)\right\|_{0} \leq & \left\|\nabla\left(u-P^{x} u\right)\left(\cdot, t_{n}\right)\right\|_{0} \\
& +C\left(\left\|\left(P^{x} u-u\right)_{t}\right\|_{0,0}^{\left(0, t_{n}\right)}+\left\|\Delta\left(P^{t} u-u\right)\right\|_{0,0}^{\left(0, t_{n}\right)}\right) .
\end{aligned}
$$

Use of the commutation and approximation properties of $P^{x}$ and $P^{t}$ in Lemmas 2.1 and 2.2 completes the proof of part (1) of the theorem.

In the same way, using the triangle inequality, we may write

$$
\left\|\left(u-u^{h k}\right)_{t}\right\|_{0,0}^{\left(0, t_{n}\right)} \leq\left\|\left(u-P^{t} P^{x} u\right)_{t}\right\|_{0,0}^{\left(0, t_{n}\right)}+\left\|\left(P^{t} P^{x} u-u^{h k}\right)_{t}\right\|_{0,0}^{\left(0, t_{n}\right)},
$$

and again using (3.4) and the error estimates in Lemma 2.2 completes the proof of part (2) of the theorem.

Our last theorem of the present section gives error estimates in the $L^{2}(\Omega)$-norm, which is the norm most usually considered in error estimates for the heat equation.

THEOREM 3.3. Suppose $\Omega$ is convex and the solution $u$ of (1.1) is such that

$$
u \in H^{q+1}\left((0, T) ; H^{1}(\Omega)\right) \text { and } u_{t} \in H^{p+1,0}(\Omega \times(0, T)),
$$

and $u(\cdot, t) \in H^{p+1}(\Omega)$ for $0 \leq t \leq T$. Let $e(\mathbf{x}, t)=u(\mathbf{x}, t)-u^{h k}(\mathbf{x}, t)$. Then the following estimate holds for $n=1,2, \ldots, N$ :

$$
\left\|e\left(\cdot, t_{n}\right)\right\|_{0} \leq C\left\{h^{p+1}\left(\left\|u\left(\cdot, t_{n}\right)\right\|_{p+1}+\left\|u_{t}\right\|_{p+1,0}^{\left(0, t_{n}\right)}\right)+k^{q+1}\|\nabla u\|_{0, q+1}^{\left(0, t_{n}\right)}\right\} .
$$

Remarks. 1. Except when $q=1$, the estimates in this theorem do not give an optimal estimate of the order of convergence. With some extra assumptions, we 
can prove superconvergence in time (i.e., an estimate of the error at $t_{n}$ proportional to $\left.h^{p+1}+k^{2 q}\right)$. However, the theorems in this section hold in more generality than those concerning superconvergence.

2. When $q=1$, the results in the above theorem should be compared to wellknown results for the standard Crank-Nicolson Galerkin method. For example, let $u_{h}^{n} \in S_{h}^{p}(\Omega)$ represent the Crank-Nicolson Galerkin estimate for $u\left(\cdot, t_{n}\right)$ with $u_{h}^{0}=P^{x} u_{0}$; the following estimate may be proved using standard methods (cf. [18, Theorem 4, page 14]):

$$
\begin{aligned}
\left\|u\left(t_{n}\right)-u_{h}^{n}\right\|_{0} \leq C\left\{h ^ { p + 1 } \left(\left\|u\left(\cdot, t_{n}\right)\right\|_{p+1}\right.\right. & \left.+\int_{0}^{t_{n}}\left\|u_{t}\right\|_{p+1} d t\right) \\
& \left.+k^{2} \int_{0}^{t_{n}}\left(\left\|u_{t t t}\right\|+\left\|\Delta u_{t t}\right\|\right) d t\right\} .
\end{aligned}
$$

The use of averages in time has allowed us to decrease the number of derivatives appearing in the norms in the term multiplying $k^{2}$.

COROLlaRY 3.4. Suppose the smoothness assumptions for Theorem 3.3 hold, and let $\Omega$ be convex. Then

$$
\left\|u-u^{h k}\right\|_{0,0}^{(0, T)} \leq C\left\{h^{p+1}\left(\sup _{0 \leq t \leq T}\|u(\cdot, t)\|_{p+1}+\left\|u_{t}\right\|_{p+1,0}^{(0, T)}\right)+k^{q+1}\|\nabla u\|_{0, q+1}^{(0, T)}\right\} .
$$

Remark. The above result is an optimal estimate in terms of order for the global $L^{2}$ error.

Proof of Corollary 3.4. The result is proved in the obvious way by using the estimate in Theorem 3.3 to estimate the error at each time step, and using the last result of Theorem 3.2 to estimate the error between steps.

Proof of Theorem 3.3. Let $\phi=T_{h}\left(P^{t} P^{x} u-u^{h k}\right)$ in the conclusion of Lemma 3.1 and use the definition of $T_{h}$. We obtain:

$$
\begin{aligned}
\int_{0}^{t_{n}} & \left(\left(P^{t} P^{x} u-u^{h k}\right)_{t}, T_{h}\left(P^{t} P^{x} u-u^{h k}\right)_{t}\right) d t+\frac{1}{2}\left\|\left(P^{x} u-u^{h k}\right)\left(\cdot, t_{n}\right)\right\|_{0}^{2} \\
& =\int_{0}^{t_{n}}\left(\left(P^{t} P^{x} u-u^{h k}\right)_{t}, T_{h}\left(P^{t} P^{x} u-u^{h k}\right)_{t}\right) \\
& \quad+\left(\left(P^{t} P^{x} u-u^{h k}\right),\left(P^{t} P^{x} u-u^{h k}\right)_{t}\right) d t \\
= & \int_{0}^{t_{n}}\left(\left(P^{t} P^{x} u-u^{h k}\right)_{t}, T_{h}\left(P^{t} P^{x} u-u^{h k}\right)_{t}\right) \\
\quad & \quad\left(\nabla\left(P^{t} P^{x} u-u^{h k}\right), \nabla T_{h}\left(P^{t} P^{x} u-u^{h k}\right)_{t}\right) d t \\
= & \int_{0}^{t_{n}}\left(\left(P^{x} u-u\right)_{t}, T_{h}\left(P^{t} P^{x} u-u^{h k}\right)_{t}\right) \\
& -\left(\Delta\left(P^{t} u-u\right), T_{h}\left(P^{t} P^{x} u-u^{h k}\right)_{t}\right) d t .
\end{aligned}
$$

Hence by the triangle inequality, the symmetry (and nonnegativity) of $T_{h}$, and the arithmetic geometric mean inequality, the following estimate holds:

$$
\begin{aligned}
\left\|\left(u-u^{h k}\right)\left(\cdot, t_{n}\right)\right\|_{0}^{2} \leq C\left(\left\|\left(u-P^{x} u\right)\left(\cdot, t_{n}\right)\right\|_{0}^{2}\right. & +\int_{0}^{t_{n}}\left(\left(P^{x} u-u\right)_{t}, T_{h}\left(P^{x} u-u\right)_{t}\right) \\
& \left.+\left(\Delta\left(P^{t} u-u\right), T_{h} \Delta\left(P^{t} u-u\right)\right) d t\right) .
\end{aligned}
$$


Hence via the a priori estimate for $T_{h}(2.6)$, we obtain

$$
\begin{aligned}
& \left\|\left(u-u^{h k}\right)\left(\cdot, t_{n}\right)\right\|_{0} \\
& \quad \leq C\left\{\left\|\left(u-P^{x} u\right)\left(\cdot, t_{n}\right)\right\|_{0}+\left(\int_{0}^{t_{n}}\left\|P^{x} u_{t}-u_{t}\right\|_{-1}^{2}+\left\|\nabla\left(P^{t} u-u\right)\right\|_{0}^{2} d t\right)^{1 / 2}\right\} .
\end{aligned}
$$

Use of error estimates in Section 2, Lemma 2.2 completes the proof.

4. Superconvergence in Time. As discussed in the introduction, the Galerkin method described in this paper is equivalent to using a diagonal Padé approximation in time when both methods are applied to the homogeneous heat equation. Thus we expect our Galerkin time stepping scheme to be superconvergent at certain special points in time. This is easy to prove in the absence of spatial discretization, and we shall consider that case first. The proof involves semidiscrete parabolic duality and stability estimates for the homogeneous semidiscrete problem. Then we shall provide a more complex proof of superconvergence for the fully discrete problem, using some techniques related to those introduced in [4] and used by Winther in his analysis of hyperbolic problems [21].

Let us first define the semidiscrete problem without spatial discretization. We seek $u^{k} \in H_{0}^{1}(\Omega) \otimes S_{k}^{q}([0, T])$ such that

$$
\int_{0}^{T}\left(u_{t}^{k}, v_{t}^{k}\right)+\left(\nabla u^{k}, \nabla v_{t}^{k}\right) d t=\int_{0}^{T}\left(f, v_{t}^{k}\right) d t \quad \forall v^{k} \in H_{0}^{1}(\Omega) \otimes S_{k}^{q}([0, T])
$$

with initial condition $u^{k}=u_{0}$ at $t=0$. Using the same arguments as for Theorem 3.1 , we can see that $u^{k}$ exists and is unique. The following error estimate shows that the solution $u^{k}$ of the semidiscrete problem is superconvergent at the time grid points $t_{n}, n=1, \ldots, N$.

THEOREM 4.1. Suppose the solution $u$ of (1.1) is such that $T^{-q+1 / 2} u \in$ $H^{0, q+1}(\Omega \times(0, T))$. Then, if $u^{k}$ solves (4.1), the following error estimate holds for $1 \leq n \leq N$ :

$$
\left\|\left(u-u^{k}\right)\left(\cdot, t_{n}\right)\right\|_{0} \leq C k^{2 q}\left\|T^{-q+1 / 2} u\right\|_{0, q+1}^{\left(0, t_{n}\right)} .
$$

Remark. For $\left\|T^{-q+1 / 2} u\right\|_{0, q+1}^{\left(0, t_{n}\right)}$ to be bounded, it is sufficient that $\|u\|_{H^{q+1}\left((0, T) ; H^{2 q-1}(\Omega)\right)}$ be bounded and $\Delta^{j} u=0$ on $\partial \Omega$ for $0 \leq j \leq q-1$ [18]. Using Eq. (1.1) at the boundary, together with the boundary data and the conditions that $\Delta^{j} u=0$ for $0 \leq j \leq q-1$, we can show that $\Delta^{k} f=0$ on $\partial \Omega$ for $0 \leq k \leq q-2$. In view of a priori estimates for parabolic problems on smooth domains [12], the need to impose boundary conditions of this type on $f$ is unexpected, but these type of constraints also appear in other work on superconvergence for parabolic problems (e.g. [6]).

Before proving this theorem we shall prove a lemma concerning the stability of the semidiscrete problem in negative spatial norms.

LEMMA 4.1. Suppose $z^{k}$ satisfies Eq. (4.1) with $f \equiv 0$ (i.e., the homogeneous semidiscrete problem). Then for $1 \leq r \leq q$ and $1 \leq n \leq N$, the following stability estimate holds with constant $C$ independent of $n$ :

$$
\sum_{m=0}^{n-1}\left(\left\|T^{r-1 / 2}\left(\frac{\partial}{\partial t}\right)^{r} z^{k}\right\|_{0,0}^{\left(t_{m}, t_{m+1}\right)}\right)^{2} \leq C\left\|z^{k}(\cdot, 0)\right\|_{0}^{2} .
$$


Proof. The proof is by induction on $r$. First, in the case $r=1$, we take $v^{k}=T z^{k}$ in (4.1), and the result follows by the same arguments as used in the proof of the stability result in Theorem 3.1 .

Now suppose the result is true for $1 \leq r \leq R-1<q$. Let $Q^{\left[t_{m}, t_{m+1}\right]}$ be the $L^{2}\left(t_{m}, t_{m+1}\right)$ projection onto $P^{q-1}\left(\left[t_{m}, t_{m+1}\right]\right)$; then taking $v^{k}=T w^{k}$ in (4.1), where $w^{k} \in H_{0}^{1}(\Omega) \otimes S_{k}^{q}([0, T])$, we find that $z^{k}$ satisfies $T z_{t}^{k}=Q^{\left[t_{m}, t_{m+1}\right]} z^{k}$ on $\left[t_{m}, t_{m+1}\right]$. Hence, using the fact that the operators in time and space commute, we obtain

$$
T^{R-1 / 2}\left(\frac{\partial}{\partial t}\right)^{R-1} z_{t}^{k}=\left(\frac{\partial}{\partial t}\right)^{R-1} Q^{\left[t_{m}, t_{m+1}\right]} T^{R-3 / 2} z^{k}
$$

Thus, if $k_{m}=t_{m+1}-t_{m}$, and using inverse estimates on a single interval in time, we find that

$$
\begin{aligned}
\left\|T^{R-1 / 2}\left(\frac{\partial}{\partial t}\right)^{R-1} z_{t}^{k}\right\|_{0,0}^{\left(t_{m}, t_{m+1}\right)} \\
\leq\left\|\left(\frac{\partial}{\partial t}\right)^{R-1}\left(Q^{\left[t_{m}, t_{m}+1\right.}-I\right) T^{R-3 / 2} z^{k}\right\|_{0,0}^{\left(t_{m}, t_{m+1}\right)} \\
\quad+\left\|\left(\frac{\partial}{\partial t}\right)^{R-1} T^{R-3 / 2} z^{k}\right\|_{0,0}^{\left(t_{m}, t_{m+1}\right)} \\
\leq C k_{m}^{-R+1}\left\|\left(Q^{\left[t_{m}, t_{m+1}\right]}-I\right) T^{R-3 / 2} z^{k}\right\|_{0,0}^{\left(t_{m}, t_{m+1}\right)} \\
\quad+\left\|\left(\frac{\partial}{\partial t}\right)^{R-1} T^{R-3 / 2} z^{k}\right\|_{0,0}^{\left(t_{m}, t_{m+1}\right)}
\end{aligned}
$$

But, by standard estimates for the $L^{2}$ projection,

$$
\begin{aligned}
& \left\|\left(Q^{\left[t_{m}, t_{m+1}\right]}-I\right) T^{R-3 / 2} z^{k}\right\|_{0,0}^{\left(t_{m}, t_{m+1}\right)} \\
& \leq C k_{m}^{R-1}\left\|\left(\frac{\partial}{\partial t}\right)^{R} T^{R-3 / 2} z^{k}\right\|_{0,0}^{\left(t_{m}, t_{m+1}\right)},
\end{aligned}
$$

and hence combining (4.3) and (4.4), we have shown that

$$
\left\|T^{R-1 / 2}\left(\frac{\partial}{\partial t}\right)^{R} z^{k}\right\|_{0,0}^{\left(t_{m}, t_{m+1}\right)} \leq C\left\|T^{R-3 / 2}\left(\frac{\partial}{\partial t}\right)^{R-1} z^{k}\right\|_{0,0}^{\left(t_{m}, t_{m+1}\right)},
$$

and induction completes the proof of the lemma.

Proof of Theorem 4.1. The proof rests on a discrete duality argument similar to those used in [16], [19], and [6]. Let $z^{k} \in H_{0}^{1}(\Omega) \otimes S_{k}^{q}([0, T])$ satisfy the semidiscrete backward heat equation

$$
\int_{0}^{T}\left(z_{t}^{k}, v_{t}^{k}\right)-\left(\nabla z^{k}, \nabla v_{t}^{k}\right) d t=0 \quad \forall v^{k} \in H_{0}^{1}(\Omega) \otimes S_{k}^{q}([0, T])
$$

together with final data $z^{k}\left(\cdot, t_{n}\right)=\left(u-u^{k}\right)\left(\cdot, t_{n}\right)$. Now let $e^{k}=P^{t} u-u^{k}$ and note that $z^{k}=e^{k}$ at $t=t_{n}$. Letting $v^{k}=T e^{k}$ in (4.5), and integrating the second term 
by parts in time, we obtain

$$
\left.\left(\nabla z^{k}, \nabla T e^{k}\right)\right|_{t_{n}}=\int_{0}^{t_{n}}\left(e_{t}^{k}, T z_{t}^{k}\right)+\left(\nabla e^{k}, \nabla T z_{t}^{k}\right) d t .
$$

Next, if we use the equation for $u$, the definition of $u^{k}$ and the definition of $P^{t}$, we can show that

$$
\begin{aligned}
\left.\left(\nabla z^{k}, \nabla T e^{k}\right)\right|_{t_{n}} & =\int_{0}^{t_{n}}\left(\left(P^{t} u-u\right)_{t}, T z_{t}^{k}\right)+\left(\nabla\left(P^{t} u-u\right), \nabla T z_{t}^{k}\right) d t \\
& =\int_{0}^{t_{n}}\left(\left(P^{t} u-u\right), z_{t}^{k}\right) d t .
\end{aligned}
$$

Finally, using the Cauchy-Schwarz inequality and the error estimates for $P^{t}$ from Lemma 2.2, we find that

$$
\begin{aligned}
\left\|\left(u-u^{k}\right)\left(\cdot, t_{n}\right)\right\|_{0}^{2} & =\int_{0}^{t_{n}}\left(\left(P^{t}-I\right) T^{-q+1 / 2} u, T^{q-1 / 2} z_{t}^{k}\right) d t \\
& \leq \sum_{m=0}^{n-1}\left\|\left(P^{t}-I\right) T^{-q+1 / 2} u\right\|_{0,-q+1}^{\left(t_{m}, t_{m+1}\right)}\left\|T^{q-1 / 2} z_{t}^{k}\right\|_{0, q-1}^{\left(t_{m}, t_{m+1}\right)} \\
& \leq C k^{2 q} \sum_{m=0}^{n-1}\left\|T^{-q+1 / 2} u\right\|_{0, q+1}^{\left(t_{m}, t_{m+1}\right)}\left\|T^{q-1 / 2} z_{t}^{k}\right\|_{0, q-1}^{\left(t_{m}, t_{m+1}\right)}
\end{aligned}
$$

Use of the result of Lemma 4.1 to estimate the term in $z^{k}$ completes the proof.

The method of proof used for Theorem 4.1 can be used to analyze the fully discrete problem. However, the final result contains quantities involving inverse powers of $T_{h}$ rather than $T$. In particular, the estimate involves $\left\|T_{h}^{-q+1 / 2} P_{x} u\right\|_{0, q+1}^{\left(t_{m}, t_{m+1}\right)}$, which seems difficult to bound independently of $h$ without some extra assumptions on the spatial grid. For this reason, when we prove error estimates for the fully discrete problem, we adopt a slightly more complex proof technique to estimate the term involving $\left(P^{t}-I\right)$. To do this, we use some auxiliary functions related to those introduced in [4] and used by Winther [21] in his analysis of hyperbolic problems. The essential features of the fully discrete proof are still that we use discrete duality and trade inverse norms in time against positive norms in space. The next theorem provides a superconvergence estimate for the fully discrete approximation.

THEOREM 4.2. Suppose that $\Omega$ is convex and that the solution $u$ of (1.1) is sufficiently smooth that

$$
u \in H^{q+1}\left((0, T) ; H^{2 q-1}(\Omega)\right) \quad \text { and } \quad u_{t} \in H^{2 q+p-1,0}(\Omega \times(0, T)) .
$$

Furthermore, suppose $u(\cdot, t) \in H^{p+1}(\Omega)$ for each $t$, and $\Delta^{j} u=0$ on $\partial \Omega$ for $0 \leq$ $j \leq q-1$. Then

$$
\begin{aligned}
& \left\|\left(u-u^{h k}\right)\left(\cdot, t_{n}\right)\right\|_{0} \\
& \quad \leq C\left\{h^{p+1}\left(\left\|u\left(\cdot, t_{n}\right)\right\|_{p+1}+\left\|u_{t}\right\|_{2 q+p-1,0}^{\left(0, t_{n}\right)}\right)+k^{2 q}\|u\|_{H^{q+1}\left(\left(0, t_{n}\right) ; H^{2 q-1}(\Omega)\right)}\right\} .
\end{aligned}
$$

Remark. When $q=1$, ‘his estimate reduces to the estimate proved in Section 3.

Before proving this result, let us define some notation. If $s \geq 0$, we define the special norm

$$
\|w\|_{s, k}^{2}=\sum_{m=0}^{N-1}\|w\|_{H^{s}\left(t_{m}, t_{m+1}\right)}^{2}
$$


and $H^{s, k}(0, T)=\left\{w \in L^{2}(0, T):\|w\|_{s, k}<\infty\right\}$. For $s$ a negative integer, we define

$$
\|w\|_{s, k}=\sup _{\phi \in H^{-s, k}(0, T)} \frac{\int_{0}^{T} w \phi d t}{\|w\|_{-s, k}} .
$$

Clearly, if $w \in H^{s}(0, T)$ with $s \geq 0$, then $\|w\|_{s, k} \leq\|w\|_{H^{s}(0, T)}$, and if $s<0$, $\|w\|_{H^{s}(0, T)} \leq\|w \mid\|_{s, k}$.

Now we introduce a special sequence of functions. Let $W^{(0)}=\left(P^{t}-I\right) u$, and let $W^{(j)} \in L^{2}(\Omega) \otimes S_{k}^{q}([0, T])$ for $j=1,2, \ldots$ be defined as the unique solution of

$$
\int_{0}^{T} W_{t}^{(j)} \phi_{t} d t=\int_{0}^{T} \Delta W^{(j-1)} \phi_{t} d t \quad \forall \phi \in S_{k}^{q}([0, T])
$$

with the initial condition $W^{(j)}(\mathbf{x}, 0)=0$ for $\mathbf{x} \in \Omega$. Of course, this construction can be extended in the $L^{2}$ sense in space if $\Delta W^{(j-1)} \in H^{0,0}(\Omega \times(0, T))$. Note that $W^{(j)}$ inherits whatever spatial smoothness is present for $\Delta W^{(j-1)}$, so that, for $s \geq 0$,

$$
\left\|W^{(j)}\right\|_{s, 0}^{\left(0, t_{n}\right)} \leq C\left\|u_{t}\right\|_{s+2 j, 0}^{\left(0, t_{n}\right)}
$$

and if $\Delta W^{(j-1)} \in H_{0}^{1,1}(\Omega \times(0, T))$ then $W^{(j)} \in H_{0}^{1,1}(\Omega \times(0, T))$. We shall need $W^{(q-1)} \in H_{0}^{1,1}(\Omega \times(0, T))$, and this holds if $\Delta^{j} u=0$ on $\partial \Omega$ for $0 \leq j \leq q-1$ and $u$ is smooth enough (e.g., $\left.u \in H^{1}\left((0, T) ; H^{2 q-1}(\Omega)\right)\right)$.

Note that if we define the function $W$ by

$$
W=\sum_{j=1}^{q-1} W^{(j)}
$$

then

$$
\int_{0}^{t_{n}}\left(\Delta\left(P^{t}-I\right) u, \phi_{t}\right) d t=\int_{0}^{t_{n}}\left(\left(W_{t}-\Delta W+\Delta W^{(q-1)}\right), \phi_{t}\right) d t
$$

for all $\phi \in L^{2}(\Omega) \otimes S_{k}^{q}\left(\left[0, t_{n}\right]\right)$. Next we shall prove two lemmas concerning the auxiliary functions $W^{(j)}$, which show that the functions are small in an appropriate sense. The lemmas correspond to the two lemmas used by Winther [21] and are proved in a similar way.

LEMMA 4.2. Let $j$ be an integer with $0 \leq j \leq q-1$. Then there is a constant $C$ such that for $0 \leq s \leq q-j-1$,

$$
\left\|W^{(j)}\right\|_{L^{2}\left(\Omega ; H^{-s, k}(0, T)\right)} \leq C k^{q+s+j+1}\|u\|_{H^{q+1}\left((0, T) ; H^{2 j}(\Omega)\right)} .
$$

Proof. The result is proved by induction on $j$. When $j=0$, the estimate holds by using the estimates for $P^{t}$ in Lemma 2.2. Next, assume the result is true for $0 \leq J-1<q-1$, and let $0 \leq s \leq q-J-1$. For $\eta \in H^{s, k}(0, T)$, define $\phi \in H^{s+1, k}(0, T)$ by $\phi_{t}=\eta$ and $\phi(T)=0$. By (4.6) and the definition of $\phi$,

$$
\begin{aligned}
\int_{0}^{T} W^{(J)} \eta d t & =\int_{0}^{T} W^{(J)} \phi_{t} d t \\
& =-\int_{0}^{T}\left(W_{t}^{(J)}-\Delta W^{(J-1)}\right)(\phi-\mu)+\Delta W^{(J-1)} \phi d t
\end{aligned}
$$


for all functions $\mu$ such that $\left.\mu\right|_{\left[t_{m}, t_{m+1}\right]} \in L^{2}(\Omega) \otimes P^{q-1}\left(\left[t_{m}, t_{m+1}\right]\right)$ when $0 \leq m \leq$ $N-1$. Hence, since $\|\phi\|_{s+1, k} \leq C\|\eta \eta\|_{s, k}$, we find that

$$
\begin{aligned}
\int_{0}^{T} W^{(J)} \eta d t \leq & C k^{s+1}\left(\left\|W_{t}^{(J)}\right\|_{L^{2}(0, T)}+\left\|\Delta W^{(J-1)}\right\|_{L^{2}(0, T)}\right)\|\eta\|_{s, k} \\
& +\left\|\Delta W^{(J-1)}\right\|_{-(s+1), k}\|\eta\|_{s, k} .
\end{aligned}
$$

Now we note that by (4.6), $\left\|W_{t}^{(J)}\right\|_{L^{2}(0, T)} \leq\left\|\Delta W^{(J-1)}\right\|_{L^{2}(0, T)}$. Furthermore, if $Z^{(j)}=\Delta W^{(j)}, 0 \leq j \leq q-1$, then $Z^{(j)}$ satisfies all the equations and estimates satisfied by $W^{(j)}$, except with $u$ replaced by $\Delta u$. Hence the induction hypothesis implies estimates for $Z^{(j)}, 0 \leq j \leq J-1<q-1$, and so

$$
\left\|\Delta W^{(J-1)}\right\|_{0,0}^{(0, T)} \leq C k^{q+J}\|\Delta u\|_{H^{q+1}\left((0, T) ; H^{2(J-1)}(\Omega)\right)} .
$$

Estimating $\left\|\Delta W^{(J-1)}\right\|_{L^{2}\left(\Omega ; H^{-(s+1)}(0, T)\right)}$ similarly by induction proves the desired estimate for $W^{(J)}$.

From Lemma 4.2 we obtain the following estimates for $W^{(j)}$.

LEMMA 4.3. Let $j$ be an integer such that $0 \leq j \leq q-1$. For each $r \geq 0$ there is a constant $C$ such that
1. $\left\|W^{(j)}\right\|_{r, 0}^{(0, T)} \leq C k^{q+j+1}\|u\|_{H^{q+1}\left((0, T) ; H^{2 j+r}(\Omega)\right)}$
2. $\max _{1 \leq n \leq N}\left\|W^{(j)}\left(\cdot, t_{n}\right)\right\|_{r} \leq C k^{2 q}\|u\|_{H^{q+1}\left((0, T) ; H^{2 j+r}(\Omega)\right)}$.

Proof. As in the proof of the previous lemma, we note that derivatives in space commute with the operations in time. Thus to estimate $\left(\partial / \partial x_{p}\right)^{i} W^{(j-1)}(\mathbf{x}, t)$, $p=1,2$, it suffices to estimate $W^{(j-1)}(\mathbf{x}, t)$ with $u$ replaced by $\left(\partial / \partial x_{p}\right)^{i} u$. With this observation, the first estimate follows directly from Lemma 4.2 with $s=0$. The second estimate is also easy to prove if $j=0$ since $P^{t} u=u$ at the time mesh points, so we need only consider the case $1 \leq j \leq q-1$, and $r=0$. But

$$
W^{(j)}\left(\mathbf{x}, t_{n}\right)=\sum_{m=0}^{n-1} \int_{t_{m}}^{t_{m+1}}\left(Q^{\left[t_{m}, t_{m+1}\right]} \Delta W^{(j-1)}\right)(\mathbf{x}, t) d t
$$

hence, using the stability of $Q^{\left[t_{m}, t_{m+1}\right]}$ in negative norms,

$$
\left\|W^{(j)}\left(\cdot, t_{n}\right)\right\|_{0} \leq C\left\|\Delta W^{(j-1)}\right\|_{L^{2}\left(\Omega ; H^{(j-q), k}(0, T)\right)},
$$

and an application of Lemma 4.2 with $u$ replaced by $\Delta u$ completes the proof.

Proof of Theorem 4.2. The proof of this theorem uses a discrete parabolic duality argument in a way similar to the proof of Theorem 4.1. Let $z^{h k} \in W^{h k}$ solve the fully discrete backward heat equation

$$
\int_{0}^{T}\left(z_{t}^{h k}, v_{t}^{h k}\right)-\left(\nabla z^{h k}, \nabla v_{t}^{h k}\right) d t=0 \quad \forall v^{h k} \in W_{h k}
$$

together with final data $z^{h k}\left(\cdot, t_{n}\right)=\left(P^{x} u-u^{h k}\right)\left(\cdot, t_{n}\right)$. Let $e^{h k}=P^{x} P^{t} u-u^{h k}$; then as in the proof of Theorem 4.1,

$$
\left\|\left(P^{x} u-u^{h k}\right)\left(\cdot, t_{n}\right)\right\|_{0}^{2}=\int_{0}^{t_{n}}\left(e_{t}^{h k}, T_{h} z_{t}^{h k}\right)+\left(\nabla e^{h k}, \nabla T_{h} z_{t}^{h k}\right) d t
$$

and so by Lemma 3.1 ,

$$
\left\|\left(P^{x} u-u^{h k}\right)\left(\cdot, t_{n}\right)\right\|_{0}^{2}=\int_{0}^{t_{n}}\left(\left(P^{x} u-u\right)_{t}, T_{h} z_{t}^{h k}\right)+\left(\Delta\left(P^{t} u-u\right), T_{h} z_{t}^{h k}\right) d t
$$


We now estimate each term on the right-hand side of (4.11). The first term is estimated as follows:

$$
\int_{0}^{t_{n}}\left(\left(P^{x} u-u\right)_{t}, T_{h} z_{t}^{h k}\right) \leq\left\|T_{h}^{1 / 2}\left(P^{x} u-u\right)_{t}\right\|_{0,0}^{\left(0, t_{n}\right)}\left\|T_{h}^{1 / 2} z_{t}^{h k}\right\|_{0,0}^{\left(0, t_{n}\right)}
$$

But by the stability estimate in Theorem 3.1,

$$
\left\|T_{h}^{1 / 2} z_{t}^{h k}\right\|_{0,0}^{\left(0, t_{n}\right)} \leq C\left\|e^{h k}\left(\cdot, t_{n}\right)\right\|_{0} .
$$

Using the a priori estimate (2.6) for $T_{h}$ and the estimates for $P^{x}$ in Lemma 2.2 completes the estimate of the first term on the right-hand side in (4.11).

To estimate the second term on the right-hand side of (4.11), we use the function $W$ defined in (4.8). By (4.9), and integration by parts in time and space,

$$
\begin{aligned}
\int_{0}^{t_{n}}\left(\Delta\left(P^{t} u-u\right), T_{h} z_{t}^{h k}\right) d t= & \int_{0}^{t_{n}}\left(\left(W_{t}-\Delta W+\Delta W^{(q-1)}\right), T_{h} z_{t}^{h k}\right) d t \\
= & \int_{0}^{t_{n}}\left(T_{h} z_{t}^{h k}, P^{x} W_{t}\right)-\left(\nabla T_{h} z^{h k}, \nabla W_{t}\right) d t \\
& +\int_{0}^{t_{n}}\left(T_{h} z_{t}^{h k},\left(I-P^{x}\right) W_{t}\right)+\left(\Delta W^{(q-1)}, T_{h} z_{t}^{h k}\right) d t \\
& +\left.\left(\nabla W, \nabla T_{h} z^{h k}\right)\right|_{t_{n}} .
\end{aligned}
$$

Hence, using the properties of $T_{h}$, then the fact that $z^{h k}$ satisfies (4.10) and the definition of $P^{x}$, we find that

$$
\begin{aligned}
\int_{0}^{t_{n}}\left(\Delta\left(P^{t} u-u\right), T_{h} z_{t}^{h k}\right) d t= & \int_{0}^{t_{n}}\left(z_{t}^{h k}, T_{h} P^{x} W_{t}\right)-\left(\nabla z^{h k}, \nabla T_{h} P_{x} W_{t}\right) d t \\
& +\int_{0}^{t_{n}}\left(T_{h} z_{t}^{h k},\left(I-P^{x}\right) W_{t}\right)+\left(\Delta W^{(q-1)}, T_{h} z_{t}^{h k}\right) d t \\
& +\left.\left(\nabla P^{x} W, \nabla T_{h} z^{h k}\right)\right|_{t_{n}} \\
= & \int_{0}^{t_{n}}\left(T_{h} z_{t}^{h k},\left(I-P^{x}\right) W_{t}\right)+\left(\Delta W^{(q-1)}, T_{h} z_{t}^{h k}\right) d t \\
& +\left.\left(P^{x} W, z^{h k}\right)\right|_{t_{n}}
\end{aligned}
$$

Applying the Cauchy-Schwarz inequality, and noting that $z^{h k}$ satisfies the a priori estimate (4.13), we obtain

$$
\begin{aligned}
& \left|\int_{0}^{t_{n}}\left(\Delta\left(P^{t} u-u\right), T_{h} z_{t}^{h k}\right) d t\right| \\
& \quad \leq\left(\left\|T_{h}^{1 / 2}\left(I-P^{x}\right) W_{t}\right\|_{0,0}^{\left(0, t_{n}\right)}+\left\|T_{h}^{1 / 2} \Delta W^{(q-1)}\right\|_{0,0}^{\left(0, t_{n}\right)}\right. \\
& \left.\quad+\left\|P^{x} W\left(\cdot, t_{n}\right)\right\|_{0}\right)\left\|e^{h k}\left(\cdot, t_{n}\right)\right\|_{0} .
\end{aligned}
$$

But by (2.6) and Lemma 4.3,

$$
\begin{aligned}
\left\|P^{x} W\left(\cdot, t_{n}\right)\right\|_{0}+\left\|T_{h}^{1 / 2} \Delta W^{(q-1)}\right\|_{0,0}^{\left(0, t_{n}\right)} & \leq C\left(\left\|W\left(\cdot, t_{n}\right)\right\|_{1}+\left\|W^{(q-1)}\right\|_{1,0}^{\left(0, t_{n}\right)}\right) \\
& \leq C k^{2 q}\|u\|_{H^{q+1}\left((0, T) ; H^{2 q-1}(\Omega)\right)}
\end{aligned}
$$


and since $W_{t} \in H_{0}^{1,1}(\Omega \times(0, T))$ (by virtue of assuming $\Delta^{j} u=0$ for $0 \leq j \leq q-1$ ), and using (4.7),

$$
\begin{aligned}
\left\|T_{h}^{1 / 2}\left(I-P^{x}\right) W_{t}\right\|_{0,0}^{\left(0, t_{n}\right)} & \leq C h^{p+1}\left\|W_{t}\right\|_{p+1,0}^{\left(0, t_{n}\right)} \\
& \leq C h^{p+1} \sum_{j=1}^{q-1}\left\|\Delta W^{(j-1)}\right\|_{p+1,0}^{\left(0, t_{n}\right)} \\
& \leq C h^{p+1}\left\|u_{t}\right\|_{p+2 q-1,0}^{\left(0, t_{n}\right)}
\end{aligned}
$$

Use of estimates (4.15) and (4.16) in (4.14) completes the estimate of the second term on the right-side of (4.11). Then use of the triangle inequality and the estimate for $P^{x}$ given by (2.16) from Lemma 2.2 complete the proof.

5. Numerical Results. In this section we shall present the results of some simple numerical experiments with (1.3). We seek to investigate two questions: first, do the higher-order in time methods possess an advantage in terms of overall computing time when compared to the lower-order methods? Second, in the theorems concerning superconvergence we require restrictive conditions on the data $f$. Are these conditions always necessary?

For simplicity we have taken $p=q$ and have performed any necessary integrations by Gauss rules (order in time depending on $q$ as discussed in the introduction, and sufficiently higher order in space).

We have only examined the case when $\Omega$ is an interval (our estimates for $\mathbf{R}^{2}$ hold for $\mathbf{R}^{1}$ ). So we take $\Omega=[0,1], u_{0}=0$, and

$$
f(x, t)=3 x \cos (3 \pi x / 2) \cos (3 t)+3 \pi \sin (3 \pi x / 2)+(3 \pi / 2)^{2} \cos (3 \pi x / 2) \sin (3 t),
$$

which results in an exact solution

$$
u(x, t)=x \cos (3 \pi x / 2) \sin (3 t) .
$$

In order to investigate the computing time question, we take $h=k$, which is the relationship suggested as best by our most general theorems on global convergence. Figure 3.1 shows the errors in the solution at $t=3$ plotted against elapsed time for the computation with various $h$ values. These experiments were performed on a SUN3/50 (with MC68881) with no other users, and so elapsed time is a good measure of cpu time for the process. The unevenness of the graphs for small time is because the process was timed to the nearest second. Figure 3.1 suggests that a given accuracy of solution is obtained more rapidly by a higher-order method (at least for the smooth solution in this example).

The second question concerns superconvergence. For these experiments we take $p=q=2$, and $k$ is chosen to be the smallest value of the time step larger than $h^{3 / 4}$, which results in $t=3$ being an integral number of time steps from $t=0$. If the superconvergence results of the previous section hold, we would expect the $L^{2}$ error at $t=3$ to be $O\left(k^{4}\right)$. Our results are shown in Figure 3.2 ; the slope of the $L^{2}$ error line is approximately 3.9 , which strongly suggests superconvergence is occurring. However, $f$ does not satisfy the conditions of our theorems in Section 4 on superconvergence, since $f$ is nonzero at $x=1$. This result suggests that it may be possible to relax some of the conditions on $f$ required by the theorems in Section 4 concerning superconvergence. 


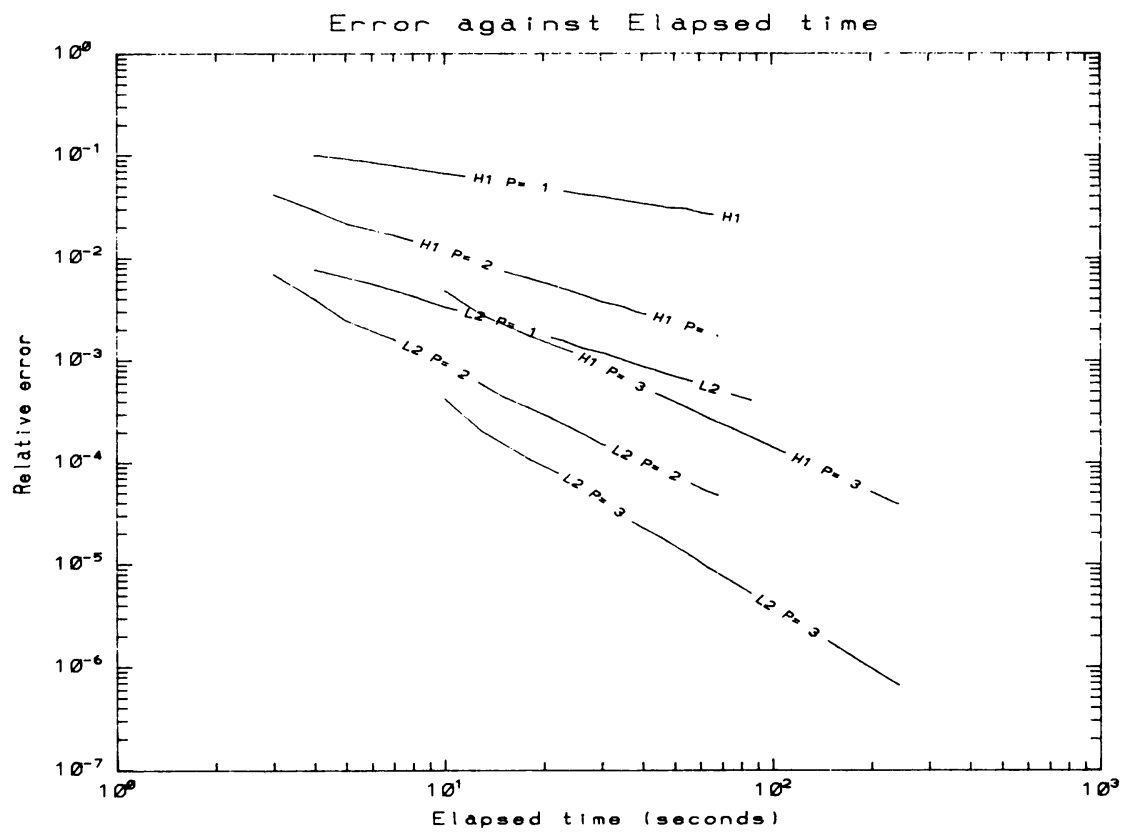

FigURE 3.1

A graph of relative error at $t=3$ against elapsed time for the computation (elapsed time was measured using the SUN time utility which measures in units of one second). Here relative error is $\left\|\left(u-u^{h k}\right)(\cdot, t)\right\|_{s} /\|u(\cdot, t)\|_{s}$ with $s=0$ or $s=1$. In this case, $p=q$ with $q=1,2$ or 3 , and $h=k$. Elapsed time includes time needed to assemble the matrices involved. These results suggest that the time necessary for a given accuracy of solution decreases as the order of the method increases (at least for this smooth example).

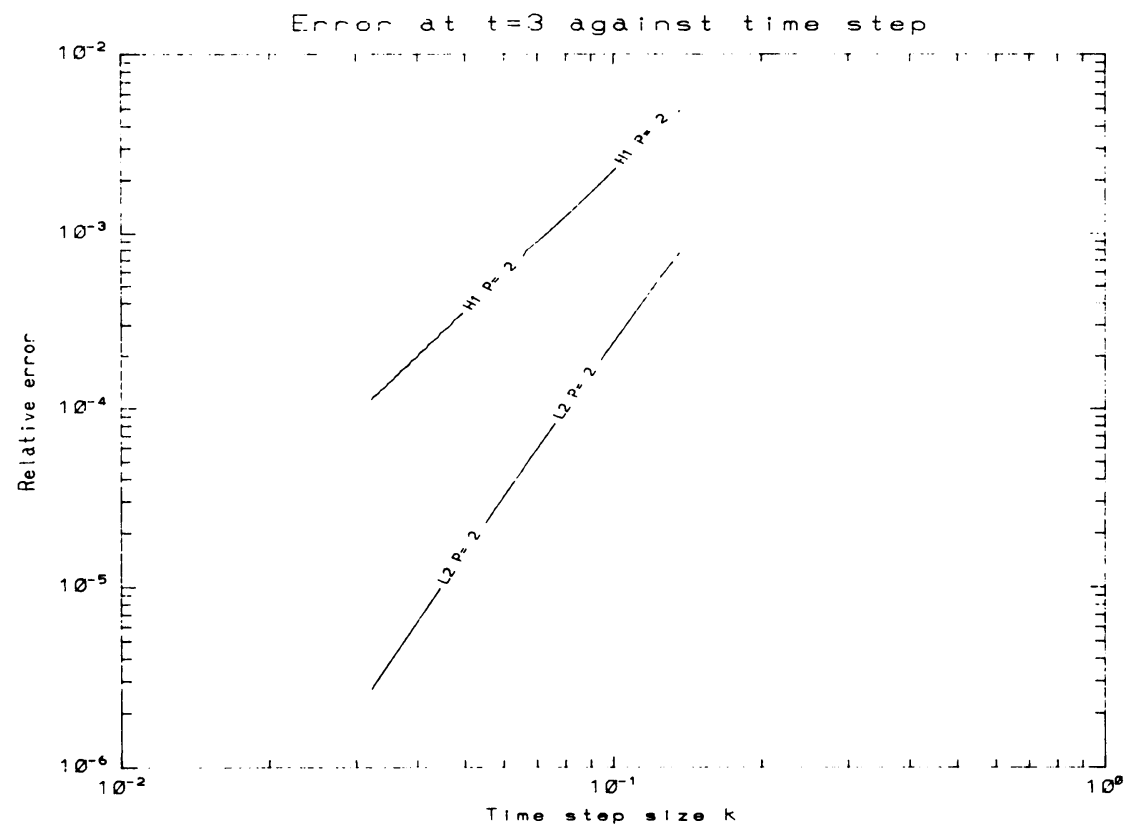

FigURE 3.2

A graph of relative error at $t=3$ against time step size $k$. Here, $p=q=2$ and $k$ is taken to be essentially $h^{3 / 4}$ (adjusted so $t=3$ is a time mesh point). The slope of the $L^{2}$ error line is about 3.9 , which suggests that superconvergence is occurring. However, the data $f$ does not satisfy the conditions of the superconvergence theorems in Section 4. 
Acknowledgments. This work was undertaken while Monk was a visitor at UMBC. The authors would also like to thank the referee for help in the preparation of this paper.

Department of Mathematical Sciences

University of Maryland, Baltimore County

Catonsville, Maryland 21228

Department of Mathematical Sciences

University of Delaware

Newark, Delaware 19716

E-mail: monk@vax1.acs.udel.edu

1. I. BABUSKA \& A. K. AzIZ, "Survey lecture on the mathematical foundations of the finite element method," in The Mathematical Foundations of the Finite Element Method with Applications to Partial Differential Equations (A. K. Aziz, ed.), Academic Press, New York, 1972, pp. 5-359.

2. P. G. Ciarlet, The Finite Element Method for Elliptic Problems, North-Holland, New York, 1980.

3. J. Douglas, JR. \& T. Dupont, Collocation Methods for Parabolic Equations in a Single Space Variable, Lecture Notes in Math., Vol. 385, Springer-Verlag, Berlin and New York, 1974.

4. J. DOUglas, JR., T. DUPONT \& M. F. WheELER, "A quasi projection analysis of Galerkin methods for parabolic and hyperbolic equations," Math. Comp., v. 32, 1978, pp. 345-362.

5. K. ERIKSSON \& C. JOHNSON, "Error estimates and automatic time step control for nonlinear parabolic problems I," SIAM J. Numer. Anal., v. 24, 1987, pp. 12-23.

6. K. ERIKSSON, C. JOHNSON \& V. THOMÉE, "Time discretization of parabolic problems by the discontinuous Galerkin method," RAIRO MAN, v. 19, 1985, pp. 611-643.

7. R. S. FALK \& G. R. RICHTER, "Analysis of a continuous finite element method for hyperbolic equations," SIAM J. Numer. Anal., v. 24, 1987, pp. 257-278.

8. B. L. HULME, "Discrete Galerkin and related one-step methods for ordinary differential equations," Math. Comp., v. 26, 1972, pp. 881-891.

9. B. L. HULME, "One-step piecewise polynomial Galerkin methods for initial value problems," Math. Comp., v. 26, 1972, pp. 415-426.

10. P. JAMET, "Galerkin-type approximations which are discontinuous in time for parabolic problems on a variable domain," SIAM J. Numer. Anal., v. 15, 1978, pp. 912-928.

11. P. JAMET, "Stability and convergence of a generalized Crank-Nicolson scheme on a variable mesh for the heat equation," SIAM J. Numer. Anal., v. 17, 1980, pp. 530-539.

12. O. A. LADYŽEnSKaja, V. A. Solonnikov \& N. N. URAL'CeVA, Linear and Quasilinear Equations of Parabolic Type, Transl. Math. Monographs, Vol. 23, Amer. Math. Soc., Providence, R. I., 1967.

13. P. LESAint \& P. A. RAVIART, "Finite element collocation methods for first order systems," Math. Comp., v. 33, 1979, pp. 891-918.

14. J. L. Lions \& E. MAGENes, Non-Homogeneous Boundary Problems and Applications I, Springer-Verlag, New York, 1972.

15. J. L. Lions \& E. MAGenes, Non-Homogeneous Boundary Problems and Applications II, Springer-Verlag, New York, 1972.

16. M. LUSKIN \& R. RANNACHER, "On the smoothing property of the Galerkin method for parabolic equations," SIAM J. Numer. Anal., v. 19, 1981, pp. 93-113.

17. S. M. NIKOL'SKII , Approximation of Functions of Several Variables and Embedding Theorems, Springer-Verlag, New York, 1975.

18. V. ThомÉE, Galerkin Finite Element Methods for Parabolic Problems, Lecture Notes in Math., Vol. 1054, Springer-Verlag, Berlin and New York, 1980.

19. V. THOMÉE, "Negative norm estimates and superconvergence in Galerkin methods for parabolic problems," Math. Comp., v. 34, 1980, pp. 93-113.

20. M. F. WhEELER, "A priori $L_{2}$ error estimates for Galerkin approximations to parabolic partial differential equations," SIAM J. Numer. Anal., v. 10, 1973, pp. 723-759.

21. R. WINTHER, "A stable finite element method for initial-boundary value problems for firstorder hyperbolic systems," Math. Comp., v. 36, 1981, pp. 65-86. 